\title{
HYPERSPECTRAL IMAGING FOR FOOD APPLICATIONS
}

\author{
Stephen Marshall, Timothy Kelman, Tong Qiao, Paul Murray, Jaime Zabalza \\ Department of Electronic and Electrical Engineering, University of Strathclyde, \\ Royal College Building, 204 George Street, Glasgow, G1 1XW, Scotland
}

\begin{abstract}
Food quality analysis is a key area where reliable, nondestructive and accurate measures are required. Hyperspectral imaging is a technology which meets all of these requirements but only if appropriate signal processing techniques are implemented. In this paper, a discussion of some of these state-of-the-art processing techniques is followed by an explanation of four different applications of hyperspectral imaging for food quality analysis: shelf life estimation of baked sponges; beef quality prediction; classification of Chinese tea leaves; and classification of rice grains. The first two of these topics investigate the use of hyperspectral imaging to produce an objective measure about the quality of the food sample. The final two studies are classification problems, where an unknown sample is assigned to one of a previously defined set of classes.
\end{abstract}

Index Terms - Signal Processing, Image Processing, Classifiers, Spectral Imaging

\section{INTRODUCTION}

Where conventional digital imaging devices capture light over one (greyscale) or three (red, green, blue) distinct portions of the electromagnetic spectrum, a hyperspectral imaging (HSI) system acquires light at hundreds or even thousands of different spectral bands. Similar to the way in which a spectrometer produces a spectrum of the light captured at a single point, an HSI system produces a spectrum for each pixel in the image. This combination of spatial and spectral detail provides significantly more information about a scene. Historically, applications of HSI have tended to be large and complex, such as remote sensing, wide area surveillance and aircraft-based systems $[1,2]$. Due to the price and size of HSI systems, it was only in these areas that the technology was a viable tool. The recent reduction in both price and size, however, has made hyperspectral imaging a more attractive option for lab based applications such as forensic science [3] and, in particular, food quality analysis [4]. In this paper, several analysis techniques are explained before the results of four different implementations of HSI in food based applications are explained. The

Thanks to Lightbody of Hamilton, Finsbury Food Group and Quality Meat Scotland results show that HSI is a useful tool in food quality analysis.

The rest of this paper is arranged as follows. In Section 2, some state-of-the-art data analysis techniques for feature extraction and data classification/prediction are discussed. Based on which, 4 case studies for food analysis are introduced in Sections 3-6, which cover shelf life estimation of baked sponges, beef quality prediction, classification of Chinese teas and rice classification using combined features. Finally, concluding remarks are presented in Section 7.

\section{DATA ANALYSIS TECHNIQUES}

This section contains overview of several different methods of data analysis ranging from feature extraction and selection through to classification.

\subsection{Feature Extraction and Dimensionality Reduction}

Conventional Principal Component Analysis (PCA) can be used as a method of both feature extraction and dimensionality reduction, making it a common technique used in HSI analysis [5]. In general, PCA transforms correlated data into uncorrelated components where only a few need to be retained to capture most of the variance within the data. By representing a dataset in a lower dimensional space in this way, faster and more efficient analysis and classification can be performed [6].

PCA operates by calculating the covariance matrix of the mean-adjusted dataset. Eigen decomposition of this covariance matrix produces its corresponding Eigenvalues and Eigenvectors. These Eigenvectors can then be used to transform the original dataset into an uncorrelated version. By sorting the Eigenvalues into descending order and discarding the smallest values, the corresponding Eigenvectors can be truncated leading to a much smaller representation of the original dataset when transformed into the new subspace.

Conventional PCA suffers from two main drawbacks when used for HSI. Firstly, the complete captured image is required before mean-adjustment can take place, preventing real-time analysis. Secondly, the calculation of the covariance matrix for the the whole dataset is a computationally intensive task. Structured Covariance PCA (SC-PCA) is one method of overcoming these problems [7]. In SC-PCA the data is 
split into sections and a partial covariance matrix is obtained for each section and then further processed as in conventional PCA. These sections can either be pixels, bands, rows or columns of an HSI dataset which correspond to the common ways in which HSI data is acquired allowing real-time processing, or in the case of already captured data, parallel processing as each section can be processed individually.

Folded-PCA [6] is a another adaptation of conventional PCA. If a hypercube has dimensions $(x, y, \lambda)$ with $x$ rows, $y$ columns and $\lambda$ bands, the data must be reshaped into a matrix of dimensions $(x \times y, \lambda)$. Each row of this matrix is treated as a vector as PCA is implemented. In Folded-PCA, each vector in this matrix is converted to a 2D matrix. Similar to SC-PCA, partial covariance matrices are calculated for each of these $2 \mathrm{D}$ sections. These can then be accumulated for Eigendecompostion and data projection. As a result, the computational cost and memory requirements are lowered. Furthermore, since local structures within the dataset can be exploited, FoldedPCA can out perform conventional PCA methods as a feature extraction method for classification problems.

Since HSI data is prone to noise, the performance of any subsequent classification can suffer. In [8] Singular Spectrum Analysis (SSA) is demonstrated to be an effective means of improving classification in a remote sensing problem. SSA operates by extraction of trends and periodic components; finding structures in short time series and envelopes of oscillation signals. In [9] SSA is extended to operate on 2D features.

\subsection{Data Prediction and Classification}

To make decisions based on captured HSI data, some form of classification is often required. Two popular methods of HSI classification are Artificial Neural Networks (ANNs) and Support Vector Machines (SVMs).

ANNs are computational models based on the operation of a biological central nervous system. ANNs consist of nodes ('neurons') linked by weighted connections. These nodes compute values based on inputs. Training of a ANN is performed by adjusting these weightings until the error between the ground truth and network output is minimised. This makes them ideal for classification problems involving HSI data [10].

SVMs are a popular classification technique for HSI problems [11]. SVMs aim to classify two-class data by finding the optimum hyperplane which separates the data. Almost always, the data is not immediately linearly separable in this way, so a technique known as the kernel trick [12] is required to map the data into a higher dimension before classification. Although a binary classifier, multiple instances can be combined in one against one or one against all fashion to achieve multi-class classification.

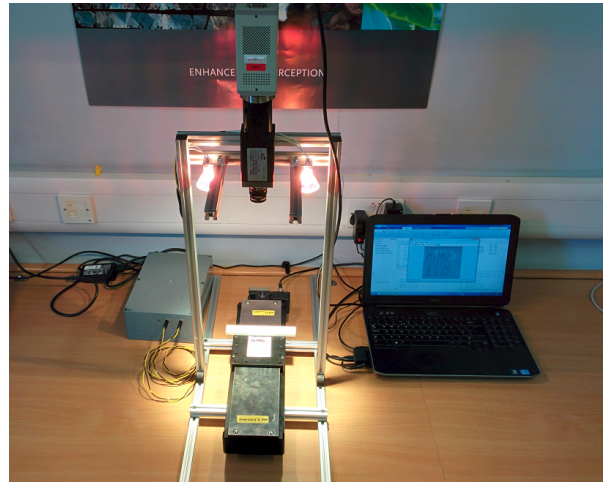

Fig. 1: VNIR HSI System

\section{SHELF LIFE ESTIMATION OF BAKED SPONGES}

In the baking industry, organoleptic testing is used to determine the eating quality of baked sponges. This testing is carried out by skilled human tasters (usually bakery staff) who eat sponges of varying ages and assign each one a score. By repeating this process over a period of time with sponges all from a single batch, the relationship between the eating quality and the age of a sponge is quantified. Based on this information, an age at which a sponge is no longer acceptable to eat can be determined, i.e. its shelf life.

This organoleptic testing process is used across the baking industry for quality analysis of a large number of products. It is, however, a very subjective test, and many external factors can affect a person's sense of taste. There are several other drawbacks such as requiring the same staff to be present for the tasting each day and a limitation on the number of products that can be tested by a single person in a single tasting session.

The aim of this study is to produce an objective, accurate and repeatable measure of the quality of a baked sponge using a visible/near infrared (VNIR) HSI system. The system used has a spectral range of $400 \mathrm{~nm}-1000 \mathrm{~nm}$ and is shown in Figure 1. By analysing the change in reflectance as a function of time at $970 \mathrm{~nm}$, a known water absorption band [13], the reduction water content (i.e. drying out) of a sponge can be estimated. This is shown in Figures 2(a) and 2(b), where the reflectance at $970 \mathrm{~nm}$ is clearly increasing for both sponge types as they get older. Although the reflectance at all wavelengths is increasing, it is more apparent around $970 \mathrm{~nm}$. By normalising and inverting these reflectance values, a decreasing score is produced. To test the correlation between this score and sponge quality, a batch of chocolate and a batch of vanilla sponges were produced. A different one of each of these sponges was imaged each day for a period of 30 days. Simultaneously, a different one of each of these sponges was organoleptically scored by a tasting panel. When compared, the scores produced by the tasting panel show a strong relationship with the scores produced from the HSI data as shown 


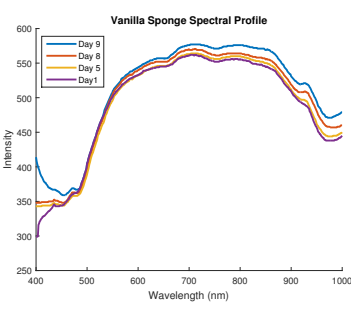

(a)

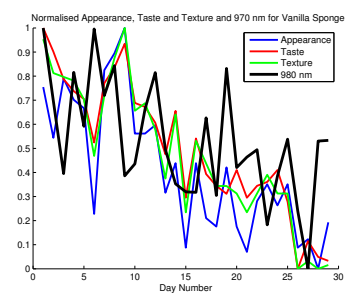

(c)

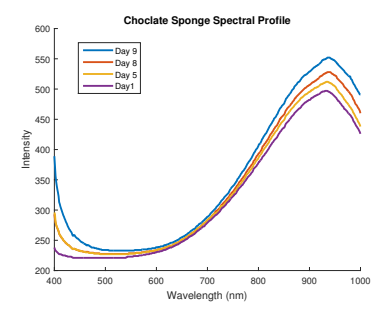

(b)

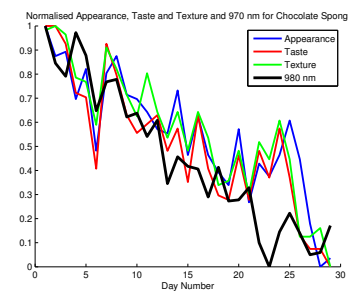

(d)
Fig. 2: HSI of Baked Sponges (a) Spectral profile ageing vanilla sponge (b) Spectral profile ageing chocolate sponge (c) HSI scores produced from reflectance at $970 \mathrm{~nm}$ compared with organoleptic data for vanilla sponge (d) HSI scores produced from reflectance at $970 \mathrm{~nm}$ compared with organoleptic data for chocolate sponge.

in Figures 2(c) and 2(d). This shows that HSI data has the potential provide an objective, non-destructive score for the quality of a baked sponge which could be used to estimate shelf life.

\section{HSI FOR BEEF QUALITY PREDICTION}

As is well known, Scottish meat has a high international reputation for its eating quality. For meat industries, identification of the quality of their products before they enter the market is crucial. The perception of consumers on meat eating quality is usually influenced by three factors: tenderness, juiciness and flavour. However, measurement of these factors is usually costly, time-consuming and destructive, which is infeasible for meat industries.

As a non-destructive approach, near-infrared spectroscopy (NIRS) has shown its potential in evaluating meat quality over the past a few decades $[14,15]$. In spite of this, due to its low spatial resolution, this technique might not be accurate when evaluating meat with non-homogeneous composition. This study looks at HSI as an alternative method for determining meat quality.

A total of 858 cattle from 4 Scottish commercial abattoirs were randomly selected from the production line. Each carcass was allowed to age for 2 days, before a piece of steak was removed for imaging, using a visible HSI system followed by an NIRS system with working wavelengths of $490-863 \mathrm{~nm}$ and $501-2200 \mathrm{~nm}$ respectively. Then each steak was halved, with one half being aged for another 5 days and the other half

\begin{tabular}{rrrrrr}
\hline System & ncal & R2cal & nval & R2val & RPDval \\
\hline NIRS & 644 & 0.42 & 214 & 0.36 & 1.22 \\
HSI & 644 & 0.7 & 214 & 0.44 & 1.38 \\
\hline
\end{tabular}

Table 1: Comparison of prediction performance for $\mathrm{pH} 14$

being aged for a further 12 days. Ultimate $\mathrm{pH}$ and slice shear force (SSF) were measured as quality references after ageing was completed.

The lean reflectance spectra were extracted from each hypercube and a median spectrum was acquired for each steak. For both HSI and NIRS spectra, reflectance was converted to absorbance to linearise the relationship between the concentration of an absorbing compound and the absorption spectrum. The whole data set was split into the training set and the testing set, where an SVM was applied to the training set to construct prediction equations. The performance was evaluated on the training set. It is proved in [15] that partial least squares regression (PLSR), which is the most common regression method researchers tend to use, underperforms SVM for beef quality evaluation using NIRS. Since SVMs are subject to the curse of dimensionality, PCA was used for both dimensionality reduction and feature extraction. For the SVM, the Radial Basis Function (RBF) kernel was selected and the optimal parameters were determined using a grid-search with 4fold cross-validation. In order to split the data set, each quality attribute was sorted in ascending order. Then every 4th sample was selected into the validation set and the rest was allocated to the calibration set. In this way, the validation set would be a representative of the calibration set, with similar average value, standard deviation and range. The prediction performance was evaluated by the coefficient of determination $\left(R^{2}\right)$ and the ratio of performance to deviation (RPD), i.e. the ratio of standard error in prediction to the sample standard deviation.

For most of the quality attributes, HSI offers higher prediction accuracies by comparing $R^{2}$ and RPD. Ultimate $\mathrm{pH}$ with 14 days ageing (pH14) is given for comparison in Table 1. Further results from this study are explained in [16] and similar research has also been done with lamb, where HSI again shows great potential for quality analysis [17].

\section{CLASSIFICATION OF CHINESE TEA SAMPLES}

In this section, a process for classification of five types of Chinese tea is documented. Due to the popularity of tea consumption and the varying prices and qualities between different brands, some form of analysis and classification is required to verify the product being sold. While conventional methods rely on subjective input from domain experts, HSI provides an objective and consistent measure, even when the examined samples appear (to the human eye) almost identical to each other. 


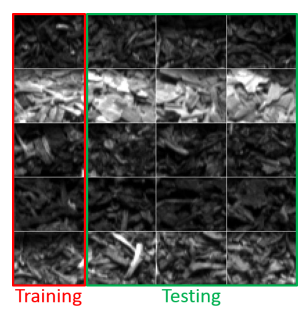

(a)

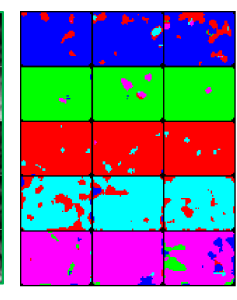

(b)
Fig. 3: Classification of tea leaves (a) All tea samples at $790 \mathrm{~nm}$ split into training and testing sets (b) Classified result after filtering.

\begin{tabular}{|c|c|c|c|c|c|c|}
\hline \multicolumn{7}{|c|}{ Predicted } \\
\hline \multirow{6}{*}{ 恶 } & & Tea 1 & Tea 2 & Tea 3 & Tea 4 & Tea 5 \\
\hline & Tea 1 & $88.94 \%$ & $0.12 \%$ & $9.59 \%$ & $0.89 \%$ & $0.46 \%$ \\
\hline & Tea 2 & $0.20 \%$ & $97.64 \%$ & $0.17 \%$ & $0.03 \%$ & $1.96 \%$ \\
\hline & Tea 3 & $0.20 \%$ & $0.00 \%$ & $97.47 \%$ & $2.26 \%$ & $0.07 \%$ \\
\hline & Tea 4 & $1.67 \%$ & $0.00 \%$ & $14.99 \%$ & $83.34 \%$ & $0.00 \%$ \\
\hline & Tea 5 & $4.47 \%$ & $4.67 \%$ & $0.57 \%$ & $0.47 \%$ & $89.82 \%$ \\
\hline
\end{tabular}

Table 2: Confusion matrix for filtered classification result

Five different tea types were imaged with a visible range (400-800 nm) HSI system, with four different samples of each tea measured, resulting in an HSI image containing 20 distinct samples. See Figure 3(a) for a greyscale representation of this image at $790 \mathrm{~nm}$.

Due to the large volume of data in an HSI image, dimensionality reduction was required to reduce the computational requirements of any subsequent processing. Using conventional PCA, the tea image was reduced from 170 bands to 10 principal components.

For classification, an ANN was used. To evaluate the performance of the classifier, training and testing data needed to be selected. Each tea type was assigned a colour for visualisation of results. To improve classification accuracy, the classification image was processed with a modal filter of size 5 pixels by 5 pixels. The final classified and filtered result is shown in Figure 3(b). Table 2 shows the confusion matrix for the filtered classification result.

From these results, it is clear that different types of tea can be classified using an HSI system even when the samples are visually similar, reducing the need for subjective testing. Furthermore, these results also show how spatial information is retained, something not possible with conventional methods of analysis. This spatial information could then be used to further improve classification.

\section{CLASSIFICATION OF DIFFERENT RICE TYPES}

In many implementations of HSI in food quality analysis, short-wave infrared (SWIR, $1000 \mathrm{~nm}-2500 \mathrm{~nm}$ ) systems tend to be used as this spectral region is rich with useful informa-

\begin{tabular}{lr}
\hline Data & Accuracy \\
\hline Spatial & $69.91 \%$ \\
Colour & $67.17 \%$ \\
Spectral & $74.27 \%$ \\
Colour + Spatial & $81.67 \%$ \\
Spectral + Spatial & $90.20 \%$ \\
\hline
\end{tabular}

Table 3: SVM Classification accuracies

tion [4]. These systems, however, are more expensive than the visible/near infrared (VNIR, $400-1000 \mathrm{~nm}$ ) systems that are available. In the SWIR region, the benefits of HSI over conventional imaging techniques are obvious. In the VNIR region, however, the advantages are less obvious. This study aims to quantify the improvements offered by a VNIR HSI system over conventional imaging systems.

The classification of four different types of rice was chosen as the subject for this study. Rice was chosen as there were several different kinds readily available and each variety showed some differences in terms of shape, size, colour and, one would expect, spectral response. A classification problem was chosen as it provides easy to understand quantifiable and therefore comparable results.

The four kinds of rice were imaged with a VNIR HSI system separately in a well-plate with 72 grains in each image. A final image of 18 grains of each rice was also captured. The true colour image was created using the spectral sensitivity function of the Nikon D70. Using the spectral difference between the rice pixels and the background pixels, masks were created for each image. Figure 4 shows a true colour representation of the rice mixture and its corresponding mask. Each grain in each mask image was then processed individually to extract three spatial features: the length of each grain, the width of each grain and its eccentricity [18].

After extraction of spatial and colour features, 5 different datasets were produced: spatial information only; colour information only; spectral information only; spatial and colour information; and spatial and spectral information.

An SVM classification was preformed using each set of 72 grains as training data and the image with 18 grains of each rice type was used as testing data. This was repeated for each of the 5 subsets explained earlier. As in Section 5, each class was assigned a colour. Figure 5 shows the classified result for the three highest scoring subsets; spectral, colour+spatial and spectral+spatial. Table 3 shows the average classification accuracies for each subset.

From the results, as expected, the highest accuracy comes from using both the spatial and spectral information. It is interesting, however, that the combination of spatial and colour information outperforms spectral information on its own. This demonstrates that a VNIR HSI system is a useful tool that when applied appropriately can improve classification results. It does not, however, negate the need for standard image processing techniques. 


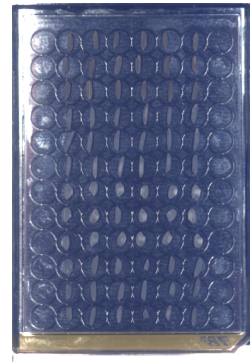

(a)

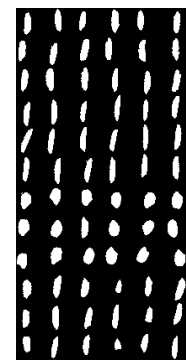

(b)
Fig. 4: Mixture of different kinds of rice (a) True colour representation (b) Corresponding extracted mask image

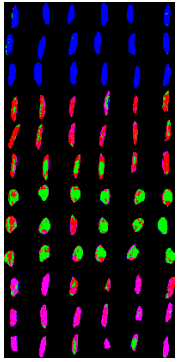

(a)

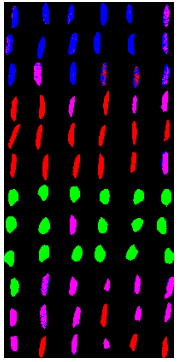

(b)

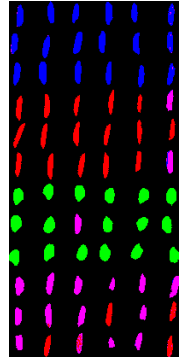

(c)
Fig. 5: SVM Classification results (a) Spectral information (b) Colour and spatial information (c) Spectral and spatial information

\section{CONCLUSION}

This paper has provided an overview of some of the popular data analysis techniques used in HSI and some of the associated recent developments. Four different studies of applications using HSI for food analysis were then presented, demonstrating the benefits offered by HSI when compared alternative methods such as organoleptic testing as well other other technologies such as spectroscopy and conventional digital imaging. Often, any measure of food quality is either subjective, e.g.based on the opinions of a skilled tasting panel, or destructive, e.g. the destructive test used to determine meat tenderness. The studies on sponges and beef have demonstrated that HSI has to the potential to either replace or augment these techniques which would result in significant benefits for the industry. The classifications of Chinese tea leaves and rice grains show how HSI can be used to differentiate between visually similar samples. This has potential to assist in a process where counterfeit products are a threat to a manufacturing business.

\section{REFERENCES}

[1] A. F. H. Goetz, G. Vane, et al., "Imaging spectrometry for earth remote sensing," Science, 1985.

[2] H. Ren and C. Chang, "Automatic spectral target recognition in hyperspectral imagery," Aerospace and Electronic Systems, IEEE Transactions on, 2003.

[3] K. Gill, J. Ren, et al., "Quality-assured fingerprint image enhancement and extraction using hyperspectral imaging," in 4th International Conference on Imaging for Crime Detection and Prevention, 2011.

[4] D. Wu and D. Sun, "Advanced applications of hyperspectral imaging technology for food quality and safety analysis and assessment: A review - part ii: Applications," Innovative Food Science \& Emerging Technologies, 2013.

[5] J. Ren, J. Zabalza, et al., "Effective feature extraction and data reduction with hyperspectral imaging in remote sensing," IEEE Signal Processing Magazine, 2014.

[6] J. Zabalza, J. Ren, et al., "Novel folded-pca for improved feature extraction and data reduction with hyperspectral imaging and sar in remote sensing," ISPRS Journal of Photogrammetry and Remote Sensing.

[7] J. Zabalza, J. Ren, et al., "Structured covariance principal component analysis for real-time onsite feature extraction and dimensionality reduction in hyperspectral imaging," Applied Optics, 2014.

[8] J. Zabalza, J. Ren, et al., "Singular spectrum analysis for effective feature extraction in hyperspectral imaging," IEEE Geoscience and Remote Sensing Letters.

[9] J. Zabalza, J. Ren, et al., "Novel two dimensional singular spectrum analysis for effective feature extraction and data classification in hyperspectral imaging," IEEE Transactions on Geoscience and Remote Sensing.

[10] P. K. Goel, S. O. Prasher, et al., "Classification of hyperspectral data by decision trees and artificial neural networks to identify weed stress and nitrogen status of corn," Computers and Electronics in Agriculture, 2003.

[11] F. Melgani and L. Bruzzone, "Classification of hyperspectral remote sensing images with support vector machines," IEEE Transactions on Geoscience and Remote Sensing, 2004.

[12] T. Hofmann, B. Schlkopf, et al., "Kernel methods in machine learning," Ann. Statist., 2008.

[13] P. J. Curran, "Remote sensing of foliar chemistry," Remote Sensing of the Environment, 1989.

[14] B Savenije, GH Geesink, JGP Van der Palen, and G Hemke, "Prediction of pork quality using visible/near-infrared reflectance spectroscopy," Meat Science, 2006.

[15] T. Qiao, J. Ren, et al., "Quantitative prediction of beef quality using visible and NIR spectroscopy with large data samples under industry conditions," Journal of Applied Spectroscopy, 2015.

[16] T. Qiao, J. Ren, et al., "Comparison between near infrared spectroscopy and hyperspectral imaging in predicting beef eating quality," in Hyperspectral Imaging Conference, Coventry, UK, 2014.

[17] T. Qiao, J. Ren, et al., "Prediction of lamb eating quality using hyperspectral imaging," in 2nd Conference on Optical Characterization of Materials, Karlsruhe, Germany, 2015.

[18] X. Ben, W. Meng, et al., "Dual-ellipse fitting approach for robust gait periodicity detection," Neurocomputing, 2012. 